\title{
Diagnostic Error Rates and Associated Factors for Lower Gastrointestinal Perforation: A Multicenter Retrospective Cross-sectional Study
}

Taku Harada ( $\sim$ hrdtaku@gmail.com )

Showa University Koto Toyosu Hospital

\section{Takashi Watari}

Shimane University Hospital

\section{Satoshi Watanuki}

Tokyo Metropolitan Tama Medical Center

Juichi Hiroshige

Showa University Koto Toyosu Hospital

\section{Seiko Kushiro}

Juntendo University

\section{Taiju Miyagami}

Juntendo University

\section{Syunsuke Shusa}

Tone Chuo Hospital

\section{Satoshi Suzuki}

Tone Chuo Hospital

Tetsuya Hiyoshi

Fukuoka University

\section{Suguru Hasegawa}

Fukuoka University

\section{Shigeki Nabeshima}

Fukuoka University

\section{Hidetoshi Aihara}

Saga Medical School Hospital

\section{Shun Yamashita}

Saga Medical School Hospital

\section{Masaki Tago}

Saga Medical School Hospital

\section{Fumitaka Yoshimura}

Yushima Clinic

Kotaro Kunitomo 
Kumamoto Medical Center

\section{Takahiro Tsuji}

Kumamoto Medical Center

\section{Masanori Hirose}

St. Marianna University School of Medicine

\section{Tomoya Tsuchida}

St. Marianna University School of Medicine

\section{Taro Shimizu}

Dokkyo Medical University Koshigaya Hospital

\section{Research Article}

Keywords: diagnostic errors, delayed diagnosis, lower gastrointestinal perforation, timely diagnosis

Posted Date: August 2nd, 2021

DOl: https://doi.org/10.21203/rs.3.rs-753657/v1

License: (c) (i) This work is licensed under a Creative Commons Attribution 4.0 International License. Read Full License 


\section{Abstract}

Lower gastrointestinal perforation is rare and challenging to diagnose timeously in the acute abdomen. However, no study has examined the frequency and associated factors of diagnostic errors related to lower gastrointestinal perforation. This large-scale multicenter retrospective study investigated the frequency of diagnostic errors and identified the associated factors. This study retrospectively analyzed data on patient factors, symptoms, situational factors, and physician factors across nine institutions between January 1, 2015 and December 31, 2019. Timely diagnosis was defined as diagnosis at the first visit to a computed tomography (CT)-capable facility or referral to an appropriate medical institution immediately following the first visit to a non-CT-capable facility; cases not meeting this definition were defined as diagnostic errors that resulted in delayed diagnosis. Of the 439 cases of lower gastrointestinal perforation identified, delayed diagnosis occurred in 138 cases (31.4\%). Multivariate logistic regression analysis showed a significant association between examination by a non-generalist and delayed diagnosis. Other factors, such as presence of fever, absence of abdominal tenderness, and unavailability of urgent radiology reports, tended to be associated with delayed diagnosis. The initial misdiagnoses mainly comprised gastroenteritis, constipation, and small bowel obstruction. In conclusion, diagnostic errors occurred in about one-third of lower gastrointestinal perforation cases.

\section{Introduction}

Recently, there has been a remarkable increase in the level of attention and development in the field of diagnostic errors, and the volume of publications have rapidly increased [1]. The frequency and impact of diagnostic errors on medical care are increasing, with diagnostic errors reported in $5 \%$ of outpatient cases [2], $10 \%$ of in-hospital deaths [3], and $7-17 \%$ of in-hospital adverse events [4, 5]. In the area of medical litigation, diagnostic errors are also reportedly the most common complaint [6]. A report by the National Academy of Medicine has identified diagnostic errors as an urgent national concern [7], and the Economic Cycle Research Institute (ECRI) has designated diagnostic errors as the number one patientsafety problem. To prevent harm associated with diagnostic errors, it is first necessary to determine their frequency and identify their associated factors [8].

Lower gastrointestinal perforation is an acute abdominal condition in which numerous bacteria from the stool spread from the small and large intestines into the abdominal cavity, causing acute diffuse peritonitis. Compared to upper gastrointestinal perforation, lower gastrointestinal perforation is less common but more severe, and is on the rise [9]. Regardless of the cause, the clinical presentation of small and large bowel perforations is relatively consistent, and management comprises resuscitation, antimicrobial therapy, and repair or reconstruction of the perforation site [10].

However, although rare, it is common to experience difficulty in diagnosing lower gastrointestinal perforation, especially timeously, in clinical practice. The preoperative diagnostic rate is approximately $10 \%$ with a high mortality rate of $30 \%[11]$. 
No studies have examined the frequency and related factors of diagnostic errors in cases with lower gastrointestinal perforation. Therefore, we conducted a large-scale multicenter retrospective study of such cases to determine the frequency of diagnostic errors and investigate their related factors.

\section{Methods}

This retrospective study was conducted at nine institutions between January 1, 2015 and December 31, 2019. Medical records were reviewed, and data were extracted for all the cases that were consistent with the diagnosis of lower gastrointestinal perforation, including: (1) those who were newly diagnosed with lower gastrointestinal perforation during the relevant period, and (2) those who had the words "free air" and "perforation" in the computed tomography (CT) report during the relevant period.

Exclusion criteria comprised cases of "perforation of the appendix," "diverticulitis," "perforation of the upper gastrointestinal," "cases in which the site of the perforation, whether upper or lower gastrointestinal, could not be determined," "cases in which the site of perforation could not be identified even though surgery was performed due to free air on CT," "gastrointestinal perforation due to endoscopy or after surgery," "cases under 15 years of age," "gastrointestinal perforation due to trauma," and "secondary perforation from mesenteric ischemia." For hospitals where electronic medical records were introduced during the study period, only cases that occurred in the period following the introduction of the electronic medical records were included.

Data collected retrospectively included patient factors (age, gender, facility resident, diabetes, dementia, psychiatric disorders, chronic laxative use, chronic analgesic use, chronic antipsychotic use, use of immunosuppressive agents [including steroids], last-minute enemas, and bedridden status), disease factors (presence of fever, abdominal pain, acute pain, abdominal tenderness, elevated C-reactive protein [CRP], and foreign body), situational factors (outpatient or inpatient, time from onset to clinical or hospital visit, facility size [greater than or less than 400 beds], teaching hospital [with or without residency programs], and availability of urgent radiology reports), characteristics of the examining physician (general practitioner, emergency physician, gastroenterologist, or other), site of perforation (the small bowel, cecum, ascending colon, transverse colon, descending colon, sigmoid colon, and rectum), and whether the perforation was caused by a foreign body or not. Due to the characteristics of the department, the general practitioners and emergency physicians were defined as generalists.

\section{Definition of timely diagnosis, delayed diagnosis, and diagnostic error}

Timely diagnosis was defined as diagnosis at the first visit to a CT-capable facility or referral to an appropriate medical institution immediately after the first visit to a non-CT-capable facility. Cases with a wrong initial diagnosis, but who were immediately referred to an appropriate medical institution and diagnosed timeously, were defined as cases with "near miss." Patients who had no evidence of perforation on initial CT, but who were finally diagnosed with lower gastrointestinal perforation after 
additional evaluation, was defined as cases with "no fault." All other cases were considered as cases with diagnostic error that resulted in delayed diagnosis.

\section{Analysis methods}

The chi-squared test or the Fisher's exact test was used to compare nominal variables. For continuous variables, $t$-tests or Wilcoxon rank-sum tests were used, as appropriate. For multiple logistic analysis, we incorporated several important factors that were likely to be significant (at $p<0.1)$ and that avoided multicollinearity, as follows: elderly patients ( $>65$ years old), visit to the health care facility after $24 \mathrm{~h}$, presence of fever, absence of abdominal tenderness, elevated CRP (above $10 \mathrm{mg} / \mathrm{L}$ ), night shift, first visit to a teaching hospital, unavailability of urgent radiology reports, first visit to the clinic, and examination by a non-generalist. Statistical analyses were performed using the EZR (Easy R) software [12]. All the tests were two-sided, with $p<0.05$ considered statistically significant.

\section{Ethics statement}

This study was approved by the ethical review board of Showa University Koto Toyosu Hospital (No.20T7044) and conducted in accordance with the Declaration of Helsinki. Written informed consent was waived owing to the retrospective study design by the ethical review board of Showa University Koto Toyosu Hospital.

\section{Results}

Of the 439 cases with lower gastrointestinal perforation that were identified in nine hospitals, 205 (46.8\%) were female and the median age was $72.21 \pm 13.92$ years. Considering the perforation sites, 230 were determined by surgery, 205 by CT, 2 by endoscopy, and 2 by contrast. Of the 439 cases, delayed diagnosis, near miss, and no fault occurred in 138 (31.4\%), 34 (7.7\%), and $17(3.9 \%)$ cases, respectively. The near-miss and no-fault cases were grouped into the timely diagnosis group. The distribution of the patient, disease, situational, and physician factors according to the two groups, namely the "Timely" and "Delayed" diagnosis groups, is shown in Table 1. Significant differences were noted between the two groups in terms of visits within $6 \mathrm{~h}$ (odds ratio [OR]: 0.47, 95\% confidence interval [Cl]: 027-0.80), visits following more than $24 \mathrm{~h}$ (OR: 1.578, 95\% Cl: 1.037-2.403), presence of fever (OR: 1.648, 95\% Cl: $1.078-$ 2.520), absence of abdominal tenderness (OR: 2.761, 95\% Cl: 1.457-5.232), elevated CRP (OR: 2.820, $95 \% \mathrm{Cl}: 1.438-5.523)$, night shift (OR: $0.560,95 \% \mathrm{Cl}: 0.345-0.909)$, visit to a teaching hospital (OR: $0.355,95 \%$ Cl: $0.234-0.537$ ), first visit to the clinic (OR: $4.061,95 \%$ Cl: $2.455-6.717$ ), unavailability of urgent radiology reports (OR: $2.306,95 \% \mathrm{Cl}$ : $1.390-3.823)$, and examination by a non-generalist (OR: 5.882, 95\% Cl: 2.796-12.356). These results are shown in Table 1.

The results of the multivariate logistic regression analysis performed using the above-mentioned nine variables are shown in Table 2. We hypothesized at the beginning of the study that delayed diagnosis was more likely to occur in the elderly. Regarding age, we conducted a multivariate analysis using an age of 65 years or older as one of the variables; the results revealed that examination by a non-generalist (OR: $3.46,95 \% \mathrm{Cl}$ : 1.13-10.60) was significantly associated with a greater likelihood of delayed diagnosis. 
Other factors, such as presence of fever (OR: 2.09, 95\% Cl: 0.90-4.83), absence of abdominal tenderness (OR: 3.27, 95\% Cl: 0.90-11.90), and unavailability of urgent radiology reports (OR: 3.15, 95\% Cl: 0.9710.20), tended to be associated with delayed diagnosis.

Of the 138 cases with delayed diagnosis, the 91 cases in which the initial diagnoses were known are shown in Table 3. The wrong initial diagnoses comprised gastroenteritis, small bowel obstruction, constipation, diverticulitis, appendicitis, and influenza in $27,13,10,5,3$, and 3 cases, respectively.

\section{Discussion}

Lower gastrointestinal perforation is one of the diagnoses of acute abdomen. Unlike in appendicitis and diverticulitis, a myriad of bacteria in the stool spread from the colon to the abdominal cavity, causing acute diffuse peritonitis, bacterial toxin absorption, and infectious shock, leading to multiple organ failure with a high mortality rate of $30-50 \%[11,13-14]$. Therefore, lower gastrointestinal perforation requires prompt diagnosis and therapeutic intervention [11]. However, it has no specific symptoms and is often misdiagnosed. Previous case series have reported that only about $10 \%$ of cases are diagnosed before surgery [11]. We conducted the first large-scale multicenter retrospective study on lower gastrointestinal perforation.

In this study, delayed diagnosis occurred in about $31 \%$ of the cases; this was less than the rates reported in previous studies [11]. In a previous study, the rate of accurate diagnosis before surgery was approximately $10 \%$ [11]; however, in this study, $60-70 \%$ of the patients had timely diagnosis. This may be because the prevalence of CT in Japan is extremely high [15] and the fact that the definition of timely diagnosis included cases in which although the initial diagnosis was wrong, the patients were referred immediately, resulting in timely diagnosis (near miss) and cases in which the immediate diagnosis was difficult (no fault).

There are no previous studies on factors associated with diagnostic errors of lower gastrointestinal perforation. Yang and Ni reported that this disease was more common in the elderly and bedridden patients, with $70 \%$ of the patients having a history of chronic constipation and $20 \%$ developing the disease after laxatives were administered [11]. Therefore, acute abdominal pain with signs of peritoneal irritation in the elderly with chronic constipation or in long-term bedridden patients should be considered as a symptom of lower gastrointestinal perforation [11]. The results of this study indicate that bedridden patients and those with a history of constipation are at risk for lower gastrointestinal perforation; however, these cases were not directly related to delayed diagnosis. Other factors such as antipsychotic use, analgesic use, use of immunosuppressive drugs including steroids, history of psychiatric disorders, and history of diabetes mellitus were also unassociated with delayed diagnosis.

In our study, the multivariate logistic regression analysis revealed that examination by practitioners other than general physicians was significantly associated with delayed diagnosis. Furthermore, presence of fever, absence of abdominal tenderness, and unavailability of urgent radiology reports tended to be associated with delayed diagnosis. 
In Japan, the training of general practitioners and emergency physicians has only just been established, and there is an urgent need to train them further. Currently, most of the primary care and emergency room services are provided by domain-specific specialists [16]. As a result, primary care and emergency medicine in Japan is provided by doctors who are not well trained in these areas. Additionally, the level of accuracy of diagnosis by gastroenterologists and non-gastroenterologists were similar in this study (OR: $1.258,95 \% \mathrm{Cl}: 0.786-2.014)$. Although this is a problem unique to Japan, the results suggest that training of the general practitioners and emergency physicians who are skilled in dealing with various symptoms decreases the risk of delayed diagnosis of lower gastrointestinal perforations and possibly of other acute illnesses.

The reason that lower gastrointestinal perforations with fever is more likely to be missed is that the presence of fever may anchor the working diagnosis to the occurrence of an infection. With some exceptions, lower gastrointestinal perforation should result in abdominal pain with signs of peritoneal irritation. In the presence of abdominal pain with peritoneal irritation signs, the differential diagnoses include appendicitis, diverticulitis, pancreatitis, or gastrointestinal perforation. Therefore, when patients have abdominal pain with peritoneal irritation signs with or without fever, the strategy to reduce delayed diagnosis is not to treat empirically with antimicrobial agents, but to perform imaging studies to confirm the diagnosis.

This study revealed that the absence of tenderness was associated with delayed diagnosis of lower gastrointestinal perforation, and the finding of an absence of abdominal tenderness in about $10 \%$ of the cases is consistent with that in Yang and Ni's study [11]. Lower gastrointestinal perforation without abdominal findings presents a diagnostic difficulty. Repeated reevaluation and follow-up of patients with abdominal pain or positive inflammatory reaction without a clear diagnosis is desirable to avoid missing these cases.

In Japan, the availability of urgent radiology reports is low, with only $26.9 \%$ cases reported in this study. Communication barriers between physicians and radiologists are due to a variety of factors, such as system factors (eg, health information technology, crowding, shift-based work, and interruptions) $[17,18]$. In our study, the results suggested that the unavailability of urgent radiology reports may be related to delayed diagnosis. To solve these problems, various factors such as training of the radiologists, improvement of the information technology systems including remote reading, and reform of the medical system are necessary.

In our study, the most common initial misdiagnoses were infectious enteritis, small bowel obstruction, constipation, diverticulitis, and appendicitis. In a previous study, upper gastrointestinal perforation was a common diagnosis, while colonic swelling, appendicitis, and pancreatitis were also reported [11]. The tendency for fatal diseases to be overlooked under the diagnosis of gastroenteritis in Japan is consistent with the finding of a previous study by Watari et al. [19]. It is understandable that constipation is a common initial diagnosis due to the presenting characteristics of the patients. The frequencies of small bowel obstruction, diverticulitis, and appendicitis among initial misdiagnoses are one of the features of 
this study. If there is small bowel obstruction with fever, increased inflammatory response, and peritoneal irritation signs, it is advisable to review the differential diagnoses. If there is abdominal pain with peritoneal irritation signs, it is advisable to request for imaging tests for a definitive diagnosis rather than simply judging the case as diverticulitis or appendicitis and treating it empirically with antimicrobial agents.

The greatest strength of this multicenter Japanese study is its large sample size; however, there are certain limitations to this study. First, the retrospective study design cannot fully exclude several common biases, including information bias, selection bias, and unexpected confounding factors. Second, we did not determine the causes of lower gastrointestinal perforation, because this disease is more common in the elderly and the bedridden, and we presumed many cases to have been managed conservatively or palliatively without surgery. In fact, only about half of the perforation sites could be identified at surgery, and many cases were treated conservatively. Additionally, previous literature has shown that the causes of non-traumatic intestinal perforation are diverse and that it is not necessary to determine them before surgery. Since the main purpose of this study was to determine the accuracy of the initial diagnosis of lower gastrointestinal perforation, and because of the abovementioned limitations, causal analysis was excluded from the design. Third, since the objective of this study was to mainly identify epidemiological characteristics of delayed diagnosis of lower gastrointestinal perforation, we did not examine the prognosis of the patients. There were many prognostic factors of acute abdomen, and most of the patients with this disease were elderly. Therefore, if we are to accurately analyze whether delayed diagnosis is associated with the prognosis, we must consider the clinical course, detailed data on patient factors and hospital factors, and even palliative cases of perforation due to advanced colorectal cancer.

In the future, we hope to conduct a retrospective analysis of error cases wherein the errors might have occurred, to determine what error patterns exist and whether they are preventable or not. In summary, delayed diagnosis of lower gastrointestinal perforation occurs in about one-third of the cases. Factors associated with delayed diagnosis probably include the presence of fever, absence of abdominal tenderness, unavailability of urgent radiology reports, and examination by a non-generalist.

\section{Declarations}

\section{Acknowledgments}

We express our appreciation to the team members from the Diagnostic Process Improvement Working Group of the Japanese Society of Internal Medicine and the Diagnostic Error Working Group of the Japanese Society of Hospital General Medicine for sharing their pearls of wisdom with us during this research. We thank Dr. Kazuya Fujiki and Prof. Hiroyuki Irie from the Department of Radiology, Faculty of Medicine, Saga University, Japan for their kind support in data acquisition. We thank Editage (www.editage.jp) for English-language editing.

\section{Author Contribution}


Taku Harada, Takashi Watari, Satoshi Watanuki and Taro Shimizu conception and design; Taku Harada, Seiko Kushiro, Taiju Miyagami, Syunsuke Syusa, Satoshi Suzuki, Tetsuya Hiyoshi, Suguru Hasegawa, Shigeki Nabeshima, Hidetoshi Aihara, Shun Yamashita, Masaki Tago, Fumitaka Yoshimura, Kotaro Kunitomo, Takahiro Tsuji, Masanori Hirose, Tomoya Tsuchida acquisition of data; Furthermore, Taku Harada and Takashi Watari have participated in writing the manuscript. All authors have reviewed and agree with the content of this manuscript.

\section{Funding}

No funding to declare.

\section{Competing Interests}

The authors declare that they have no conflict of interest.

\section{Data availability}

The datasets generated during and/or analyzed during the current study are available from the corresponding author on reasonable request.

\section{References}

1. Graber, M. L. Progress understanding diagnosis and diagnostic errors: thoughts at year 10. Diagnosis (Berl), 7, 151-159 (2020).

2. Singh, H., Meyer, A. N. \& Thomas, E. J. The frequency of diagnostic errors in outpatient care: estimations from three large observational studies involving US adult populations. B.M.J. Qual. Saf, 23, 727-731 (2014).

3. Shojania, K. G., Burton, E. C. \& McDonald, K. M. \&Goldman, L. Changes in rates of autopsy-detected diagnostic errors over time: a systematic review. J.A.M.A, 289, 2849-2856 (2003).

4. Thomas, E. J. et al. Incidence and types of adverse events and negligent care in Utah and Colorado. Med. Care, 38, 261-271 (2000).

5. Brennan, T. A. et al. Incidence of adverse events and negligence in hospitalized patients. Results of the Harvard Medical Practice Study I. N. Engl. J. Med, 324, 370-376 (1991).

6. Saber Tehrani, A. S. et al. B.M.J. Qual. Saf, 22, 672-680 https://doi.org/10.1136/bmjqs-2012-001550 (2013). 25-Year summary of US malpractice claims for diagnostic errors 1986-2010: an analysis from the National Practitioner Data Bank

7. Balogh, E., Miller, B. \& Ball, J. Improving diagnosis in health care (National Academies Press, Washington, DC, 2015).

8. Economic Cycle Research Institute.Top 10 patient safety concerns 2020. (2020) https://www.ecri.org2020.9. Accessed 08 May 2021 
9. Lanas, A. et al. Time trends and impact of upper and lower gastrointestinal bleeding and perforation in clinical practice. Am. J. Gastroenterol, 104, 1633-1641 (2009).

10. Brown, C. V. Small bowel and colon perforation. Surg. Clin. North Am, 94, 471-475 (2014).

11. Yang, B. \& Ni, H. K. Diagnosis and treatment of spontaneous colonic perforation: analysis of 10 cases. World J. Gastroenterol, 14, 4569-4572 (2008).

12. Kanda, Y. Investigation of the freely available easy-to-use software 'EZR' for medical statistics. Bone Marrow Transplant, 48, 452-458 (2013).

13. Kasahara, Y., Matsumoto, H., Umemura, H., Shirafa, S. \& Kuyama, T. Idiopathic perforation of the sigmoid colon in Japan. World J. Surg, 5, 125-130 (1981).

14. Serpell, J. W. \& Nicholls, R. J. Stercoral perforation of the colon. Br. J. Surg, 77, 1325-1329 (1990).

15. OECD. Computed tomography (CT) scanners. https://data.oecd.org/healtheqt/computedtomography-ct-scanners.htm. Accessed 30 June 2021(2021).

16. Higashi, H., Takaku, R., Yamaoka, A., Lefor, A. K. \& Shiga, T. The dedicated emergency physician model of emergency care is associated with reduced pre-hospital transportation time: a retrospective study with a nationwide database in Japan. PLoS One, 14, e0215231 (2019).

17. Fairbanks, R. J., Guarrera, T. K., Bisantz, A. B., Venturino, M. \& Westesson, P. L. Opportunities in IT support of workflow \& information flow in the emergency department digital imaging process. Proc Hum Factors Ergon. Soc. Annu. Meet, 54, 359-363 (2010).

18. Enayati, M. et al. Monitoring diagnostic safety risks in emergency departments: protocol for a machine learning study. J.M.I.R. Res. Protoc, 10, e24642 (2021).

19. Watari, T. et al. Factors and impact of physicians' diagnostic errors in malpractice claims in Japan. PLoS One, 15, e0237145 (2020).

\section{Tables}

Due to technical limitations, table 1, 2, 3 are only available as a download in the Supplemental Files section.

\section{Supplementary Files}

This is a list of supplementary files associated with this preprint. Click to download.

- Table1.xlsx

- Table2.xlsx

- Table3.xlsx 